\title{
III. DEEP SEA DRILLING PROJECT LEG 50 LABORATORY PHYSICAL-PROPERTY METHODS
}

\author{
Robert E. Boyce, Deep Sea Drilling Project, Scripps Institution of Oceanography, La Jolla, California
}

\section{INTRODUCTION}

During DSDP-IPOD Leg 50, all cores were processed through a wet-bulk-density measuring device, the Gamma Ray Attenuation Porosity Evaluator (GRAPE), which continuously scanned the core along its entire length. In addition, undisturbed samples were selected for discrete measurements of compressional-sound velocity, wet-bulk density, water content ${ }^{1}$ of the wet sample, and porosity. A few samples for heat conductivity and vane shearstrength measurements were also taken. The definitions, methods, calibrations, and calculations of these properties are discussed below.

Wet-bulk density is the ratio of the mass of a watersaturated geologic sample to its volume, in units of grams per cubic centimeter $\left(\mathrm{g} / \mathrm{cm}^{3}\right)$. Wet-water content is the ratio of the weight of sea water in a geologic sample to the weight of the saturated geologic sample, expressed as a percentage. Porosity is the ratio of the volume of pore space in a geologic sample to the volume of the saturated sample, usually reported in per cent or as a decimal fraction.

Sound velocity is the compressional-sound velocity at $400 \mathrm{kHz}$ through saturated geologic material, reported in units of kilometers per second.

Heat conductivity is the quantity of heat per second which flows between opposite faces of a cube which differ in temperature by $1^{\circ} \mathrm{C}$. Heat conductivity in a $1-\mathrm{cm}$ cube is a coefficient in units of $\left(\mathrm{cal} . \times 10^{3}\right) /\left(\mathrm{cm}-{ }^{\circ} \mathrm{C}\right.$-s $)$.

Vane shear strength is the shearing force $(\mathrm{kg})$ per unit area $\left(\mathrm{cm}^{2}\right)$ for water-saturated clayey sediment. It represents primarily clayey cohesion of the Leg 50 material, and not (theoretically) friction of the coarser grains.

\section{Physical Condition of Samples}

We attempted to obtain an undisturbed sample. Core disturbance is a serious problem in the upper 200 meters of the hole where the sediments are soft. Because the drill bit is $25 \mathrm{~cm}$ in diameter and has a 6-cm-diameter coring hole, most soft sediments are squirted into the core barrel and are extremely disturbed.

Because of this core disturbance, we did not take many discrete samples for physical-property measurements in the upper part of the hole, but we subjected all the cores to the continuous-analog GRAPE. Also, vane shear strength and heat conductivity were measured on only a small number of "semi-soft" samples in the up-

\footnotetext{
${ }^{1}$ All water-content measurements were done by the DSDP technical group.
}

per part of the hole, because the samples were either soft and disturbed, or undisturbed but too stiff for shearvane analysis.

The criterion for selecting an undisturbed sample is the presence of undistorted bedding or laminae. The obvious problem with this criterion is that a completely homogeneous soft-sediment core would not be sampled even if it were undisturbed.

We attempted to keep all samples saturated with water. The sediment cores were sealed in the original plastic liner and kept in the laboratory for 4 hours to allow them to approach room temperature (within 1 or $2{ }^{\circ} \mathrm{C}$ ). (This is necessary to obtain valid velocity data.) The cores were then split lengthwise with a cheesecutter, if soft, or with a circular diamond saw (with fresh water), if hard.

The sample used to determine sound velocity was cut from the core immediately after the core was split, so that the sample would not desiccate. The sample was trimmed with knife or diamond circular saw so that a velocity could be measured perpendicular to and parallel to the bedding. The sample was smoothed with a knife or file, squirted with fresh water, wrapped in a film of plastic, and sealed, with a wet sponge, in a rectangular box. The sample was maintained in this condition until physical-property measurements were made.

At the time physical-property measurements were begun, the sample was cleaned and scraped for a smearslide preparation; a piece ( $\leq 1 \mathrm{~g})$ was removed to measure its wet-water content aboard ship (Cahn balance). The velocity of the remaining sample was measured parallel to and perpendicular to bedding. The sample was then processed for wet-bulk density by the static 2-minute-count GRAPE technique (discussed below). In some cases half the sample was saved for a wet-watercontent measurement on shore which would be compared to the shipboard value.

\section{WET-WATER CONTENT}

\section{Shipboard Technique}

The sample we used to determine wet-water content of the wet sample aboard ship weighed less than 1 gram. Each shipboard weighing is accurate to \pm 1 per cent; therefore, each water-content value is accurate to \pm 2.5 per cent absolute units. The wet-water-content sample was wrapped in a plastic film and placed with damp paper tissue in a tape-sealed $10-\mathrm{cm}^{3}$ vial. This vial was then stored in a refrigerator (above $0^{\circ} \mathrm{C}$ ) until the actual measurements were performed. Water contents of wet samples are calculated as follows: 


$$
\text { Wet-water content }(\%)=
$$

$100 \times[$ (weight of wet sediment) - (weight dry sediment + salt $)]$

(weight of wet sediment)

We assume salt corrections for a median salinity of 45 per mill because at these sites the interstitial-water salinities varied from 35 to 60 per mill. The salt correction (1.0471) is calculated according to Hamilton's (1971) technique as described in Boyce (1976):

Salt-corrected water content of wet sample $=$ 1.0471 (wet-water content without salt correction)

These shipboard measurements were made with preweighed aluminum dishes, and measurements were accordingly corrected.

\section{Shore Technique}

The wet-water content determined on shore was also calculated from Equations 1 and 2, but the sample was weighed in pre-weighed glass bottles with bevel-edged caps. The bottle and cap were secured with several rubber bands and sealed with rubber cement. These weighing bottles were stored with a wet sponge in a heatsealed plastic bag. This plastic bag was placed in two additional heat-sealed plastic bags before being shipped to the DSDP laboratory at Scripps Institution of Oceanography.

At DSDP the rubber bands and rubber cement were completely removed from the bottles. The wet sample in the capped weighing bottle was then weighed, dried with the cap ajar at $110^{\circ} \mathrm{C}$ for 24 hours, cooled in a desiccator for at least 2 hours, and weighed again.

Precision of this method is within 1 per cent. The method was tested with three samples which had been stored for 1 month. The 50 to 60 gram weights of the wet sample and weighing bottle were reproduced within \pm 0.002 gram (the results are given in Table 1). These samples also traveled across the U.S. and back during the storage period.

The wet-water-content determinations made on shore are compared to the shipboard determinations in Table 2. Most of these shipboard wet-water-content data are within \pm 2.4 per cent (absolute units) of the values determined on shore. The average water contents differ by 1 per cent absolute $(7.08 \%$ for shore-based versus $6.06 \%$ for the shipboard determination). If we delete a single atypical sample $(416 \mathrm{~A}-46-1,75 \mathrm{~cm})$, which yielded a large (3.9 per cent) absolute difference between the ship and shore determinations, then the average of determinations made aboard ship $(6.40 \%)$ differs by only

TABLE 1

Weights of Bottle, Cap, and Wet Sample Before and After Storage for One Month

(cup sealed with rubber cement)

\begin{tabular}{lccc}
\hline & Bottle A & Bottle B & Bottle C \\
\hline 23 December 1976 & 57.5991 & 53.0292 & 63.1856 \\
28 January 1976 & 57.5992 & 53.0293 & 63.1834 \\
\hline
\end{tabular}

TABLE 2

Shore and Shipboard Wet-Water-Content Determinations (1.047 Salt Correction) for Hole 416A

\begin{tabular}{|c|c|c|}
\hline $\begin{array}{c}\text { Sample } \\
\text { (Interval in } \mathrm{cm} \text { ) }\end{array}$ & $\begin{array}{c}\text { Shore Wet- } \\
\text { Water Content } \\
(\%)\end{array}$ & $\begin{array}{l}\text { Ship Wet- } \\
\text { Water Content a } \\
\text { (\%) }\end{array}$ \\
\hline $\begin{array}{l}30-1,78.0 \\
37-2,3.0 \\
37-2,55.5 \\
37-4,18.0 \\
37-4,56.5 \\
37-4,70.5 \\
46-1,7.0 \\
46-1,75.0 \\
46-1,83.0 \\
46-2,1.0\end{array}$ & $\begin{array}{c}10.20 \\
7.17 \\
11.08 \\
9.97 \\
6.20 \\
6.70 \\
6.57 \\
(6.89) ? \\
3.73 \\
2.24\end{array}$ & $\begin{array}{c}9.33 \\
4.77-6.23 \mathrm{~b} \\
9.37-13.10^{\mathrm{b}} \\
10.68-10.94 \mathrm{~b} \\
4.67-5.43^{\mathrm{b}} \\
5.47-7.58 \mathrm{~b} \\
4.84-5.51^{\mathrm{b}} \\
(2.97) ? \\
2.95-3.86^{\mathrm{b}} \\
0.63\end{array}$ \\
\hline Mean & $\mathrm{an}=7.08$ & 6.06 \\
\hline $\begin{array}{l}\text { If we delete sample } \\
\text { number } 416 \mathrm{~A}-46-1 \text {, } \\
75 \mathrm{~cm} \text {, the mean }\end{array}$ & $=(7.10)$ & (6.40) \\
\hline
\end{tabular}

a 0.5 per cent (absolute) from the average of the determinations $(7.10 \%)$ made on shore. Only a small number of samples (9) was used in this comparison; thus the variation is not significant.

\section{WET-BULK DENSITY BY GRAPE TECHNIQUES}

\section{Introduction}

During Leg 50 we used the GRAPE device which was developed and described by Evans (1965) and modified as discussed by Boyce (1976). See Boyce (1976) for calibration, discussion, interpretation precautions, and derivation of the computer calculations made on shore and used here.

\section{Analog GRAPE}

The magnetic-tape system for the analog GRAPE device was not available during Leg 50. In addition, we could not use the calipers, and diameters were measured by hand and from the core photographs. All diameter adjustments to resolve the geometric problems discussed in Boyce (1976) were applied. We present here only information sufficient to show how, and by which equations given in Boyce (1976), the data were calculated.

The analog GRAPE data include for each core (1) a 6.61-cm-diameter aluminum standard in liner, (2) a 2.54-cm-diameter aluminum standard in liner, and (3) the actual core in liner. These data were processed on the ship through Equation 15 of Boyce (1976), assuming a constant attenuation coefficient of $0.1 \mathrm{~cm}^{2} / \mathrm{g}$ and a constant thickness of $6.61 \mathrm{~cm}$ for both standards and the core sample. The resulting data were then processed through Equation 25 of Boyce (1976) on shore, by computer, using the following parameters: the corrected density of the $6.61-\mathrm{cm}$ aluminum standard $\left(\varrho_{\mathrm{ac}}\right)$ is 2.6 $\mathrm{g} / \mathrm{cm}^{3}$, and the assigned density of the $2.54-\mathrm{cm}$ 
aluminum standard is $1.00 \mathrm{~g} / \mathrm{cm}^{3}$. The latter is substituted for the corrected density $\left(\varrho_{\mathrm{fc}}\right)$ of the water standard. The Evans (1965) "corrected" wet-bulk density $\left(e_{b c}\right)$, obtained from Equation 25, is then adjusted for (1) the diameter of core (investigators may obtain diameters from the core photographs or the DSDP repository); (2) the "corrected density" of the air, water, sediment, or breccia around the core (see Table 3); and (3) whether or not the diameter of the core is aligned to the gamma-ray beam (see Table 3 ). These geometric adjustments are applied by placing $\varrho_{b c}$ in Equations 32 through 36 of Boyce (1976). The new, geometrically adjusted $\varrho_{b c}$ is processed through Equation 27 of Boyce (1976) to adjust for the anomalous $1.128 \mathrm{~g} / \mathrm{cm}^{3}$ "corrected" density of sea water. The following parameters are used in Equation 27: grain density $\left(\varrho_{\mathrm{g}}\right)=2.7 \mathrm{~g} / \mathrm{cm}^{3}$, "corrected" grain density $\left(\varrho_{\mathrm{gc}}\right)=2.7 \mathrm{~g} / \mathrm{cm}^{3}$, fluid density $\left(\varrho_{\mathrm{f}}\right)=1.025 \mathrm{~g} / \mathrm{cm}^{3}$, and "corrected" fluid density $\left(\varrho_{\mathrm{fc}}\right)=1.128 \mathrm{~g} / \mathrm{cm}^{3}$. The final wet-bulk density $\left(\varrho_{\mathrm{b}}\right)$ derived from Equation 27 is the "true" wet-bulk density ( $\pm 11 \%$ per each $3.17 \mathrm{~mm}$ length along the core) and assumes that all mineral grains have (1) a quartz attenuation coefficient, (2) a true grain density of $2.7 \mathrm{~g} / \mathrm{cm}^{3}$, and (3) a corrected grain density of $2.7 \mathrm{~g} / \mathrm{cm}^{3}$.

\section{Static 2-Minute GRAPE}

The static mode of the GRAPE allows individual samples to be counted for a 2 -minute period with a precision of \pm 2 per cent. These samples are labeled "GRAPE Special 2-Minute Count Wet-Bulk Density."

Numerous quartz and different-diameter aluminum standards were run prior to coring at Hole 415. A set of $2.54-\mathrm{cm}$ and $6.61-\mathrm{cm}$ aluminum standards were processed with each data sheet. The standards were used to calculate the apparent quartz attenuation coefficient $\left(\mu_{\mathrm{a}}\right)$ using Equations 22 and 23 of Boyce (1976). The quartz and aluminum coefficients (averages of 9 to 12 measurements each) matched within $0.0007 \mathrm{~cm}^{2} / \mathrm{g}$. The results of the aluminum-standard measurements, which were run before and between cores, are listed in Tables 4 and 5. The average coefficients in Table 4 are applied to the 2-minute GRAPE data from Hole 415 to Core 16 in Hole 416A; the average coefficients in Table 5 are applied to data acquired from Hole 416A, Cores 17 through 57 . These two sets of data were required because we repaired and re-calibrated the GRAPE at sea.

The data in these tables indicate that no significant difference $(0.3 \%$ to $0.4 \%)$ exists in "apparent" quartz attenuation coefficients, $\mu_{\mathrm{a}}$, for different-diameter standards, as discussed in Boyce (1976). Therefore, we calculated our 2-minute count GRAPE data somewhat differently from Boyce (1976), in that we used Equation 24 of Boyce (1976), to calculate true density and did not need to determine $\mu_{\mathrm{a}}$ by a linear interpolation between $6.61-\mathrm{cm}$ aluminum standard and $2.54-\mathrm{cm}$ aluminum standard. Instead we used a constant value of 0.1028 $\mathrm{cm}^{2} / \mathrm{g}$ (the average $\mu_{\mathrm{a}}$ in Table 4) for samples from Site 415 and from Hole 416A through Core 16, and a con-
TABLE 3

Geometric Condition of Leg 50 Cores when Processed Through the GRAPE ${ }^{a}$

\begin{tabular}{|c|c|c|c|}
\hline Core & Section & $\begin{array}{c}\text { Interval } \\
(\mathrm{cm})\end{array}$ & Geometric Condition $\mathrm{b}$ \\
\hline \multicolumn{4}{|c|}{ Hole 415} \\
\hline $\begin{array}{l}1-3 \\
4-5\end{array}$ & & & $\begin{array}{r}A \\
C-1\end{array}$ \\
\hline
\end{tabular}

Hole 415A

$\begin{array}{rrrr}5 & 1 & 0-30 & \text { C-4 } \\ 5 & 1 & 30-52 & \text { C-1 } \\ 5 & 1 & 52-95 & \text { A } \\ 5 & 1 & 95-101 & \text { C-3 } \\ 5 & 1 & 101-150 & \text { A } \\ 6 & 1 & 0-140 & \text { C-1 } \\ 6 & 1 & 140-150 & \text { A } \\ 6 & 2 & 0-95 & \text { C-1 } \\ 6 & 2 & 95-105 & \text { C-4 } \\ 6 & 2 & 105-150 & \text { C-1 } \\ 8 & 1 ; 2 & & \text { C-4 } \\ 9 & 1 & 0-15 & \text { C-4 } \\ 9 & 1 & 15-140 & \text { C-1 } \\ 9 & 1 & 140-150 & \text { C-4 } \\ 9 & 2 & 0-10 & \text { C-4 } \\ 9 & 2 & 10-50 & \text { C-1 } \\ 9 & 2 & 50-65 & \text { C-4 } \\ 9 & 2 & 65-150 & \text { C-1 } \\ 9 & 3 & 0-15 & \text { C-1 } \\ 9 & 3 & 15-27 & \text { C-4 } \\ 9 & 3 & 27-150 & \text { C-1 } \\ 9 & 4 ; 5 & & \text { C-1 } \\ 10-14 & & & \text { C-4 }\end{array}$

Hole 415B

2

C-1

Hole 416A

\begin{tabular}{llcl}
1 & 1 & & C-3 \\
1 & 2 & & A \\
2 & 1 & $0-22$ & C-3 \\
2 & 1 & $22-150$ & C-1 \\
2 & 2 & & C-1 \\
2 & 3 & $0-26$ & C-1 \\
2 & 3 & $26-35$ & C-4 \\
2 & 3 & $35-110$ & C-1 \\
2 & 3 & $110-117$ & C-4 \\
2 & 3 & $117-150$ & C-1 \\
2 & 4 & $0-20$ & C-1 \\
2 & 4 & $20-30$ & C-4 \\
2 & 4 & $30-150$ & C-1 \\
3 & 1 through 3 & $0-10$ & C-2 \\
6 & 1 & C-3 \\
6 & 1 & $10-70$ & C-1 \\
6 & 1 & $70-100$ & C-4 \\
6 & 1 & $100-150$ & A \\
6 & 2 & $0-22$ & C-1 \\
6 & 2 & $22-82$ & C-4 \\
6 & 2 & $82-93$ & C-1 \\
6 & 2 & $93-115$ & C-4 \\
6 & 2 & $115-150$ & C-1 \\
6 & 3 & $0-30$ & C-1 \\
6 & 3 & $30-37$ & C-4 \\
\hline
\end{tabular}


TABLE 3 - Continued

\begin{tabular}{|c|c|c|c|}
\hline Core & Section & $\begin{array}{c}\text { Interval } \\
(\mathrm{cm})\end{array}$ & Geometric Condition $b$ \\
\hline \multicolumn{4}{|c|}{ Hole $416 \mathrm{~A}$} \\
\hline $\begin{array}{l}6 \\
6 \\
6 \\
6 \\
6\end{array}$ & $\begin{array}{l}3 \\
3 \\
3 \\
3 \\
3\end{array}$ & $\begin{array}{c}37-68 \\
68-84 \\
84-99 \\
99-111 \\
111-150\end{array}$ & $\begin{array}{l}C-1 \\
C-4 \\
C-1 \\
C-4 \\
C-1\end{array}$ \\
\hline $\begin{array}{l}6 \\
7 \\
7 \\
7 \\
7\end{array}$ & $\begin{array}{l}4 \\
1 \\
2 \\
2 \\
2\end{array}$ & $\begin{array}{c}0-80 \\
80-100 \\
100-150\end{array}$ & $\begin{array}{l}\text { A } \\
\text { C-1 } \\
\text { C-1 } \\
\text { C-4 } \\
\text { C-1 }\end{array}$ \\
\hline $\begin{array}{l}7 \\
7 \\
8 \\
8 \\
9\end{array}$ & $\begin{array}{l}\quad 3 \\
\quad 3 \\
1 \text { through } 5 \\
6 \text { through } 7 \\
1 \text { through } 4\end{array}$ & $\begin{array}{c}0-64 \\
64-150\end{array}$ & $\begin{array}{l}\text { C-1 } \\
\text { C-4 } \\
\text { A } \\
\text { C-3 } \\
\text { C-1 }\end{array}$ \\
\hline $\begin{array}{l}9 \\
9 \\
9 \\
9 \\
9\end{array}$ & $\begin{array}{l}5 \\
5 \\
5 \\
5 \\
6\end{array}$ & $\begin{array}{c}0-25 \\
25-35 \\
35-105 \\
105-150 \\
0-7\end{array}$ & $\begin{array}{l}\text { C-1 } \\
C-4 \\
\text { C-1 } \\
\text { C-4 } \\
\text { C-3 }\end{array}$ \\
\hline $\begin{array}{r}9 \\
10 \\
11 \\
11 \\
11\end{array}$ & $\begin{array}{c}6 \\
1 \text { through } 3 \\
4 \\
4\end{array}$ & $\begin{array}{l}7-150 \\
0-95 \\
95-110\end{array}$ & $\begin{array}{l}\text { C-1 } \\
\text { C-1 } \\
\text { C-1 } \\
\text { C-1 } \\
\text { C-4 }\end{array}$ \\
\hline $\begin{array}{l}11 \\
11 \\
11 \\
11 \\
11\end{array}$ & $\begin{array}{l}4 \\
5 \\
5 \\
5 \\
5\end{array}$ & $\begin{array}{c}110-150 \\
0-65 \\
65-126 \\
126-142 \\
142-150\end{array}$ & $\begin{array}{l}\text { C-1 } \\
\text { C-1 } \\
\text { C-4 } \\
\text { C-1 } \\
\text { C-4 }\end{array}$ \\
\hline $\begin{array}{l}11 \\
12 \\
12 \\
12 \\
12\end{array}$ & $\begin{array}{l}6 \\
1 \\
2 \\
2 \\
2\end{array}$ & $\begin{array}{r}0-17 \\
17-36 \\
36-84\end{array}$ & $\begin{array}{l}\text { C-1 } \\
\text { C-1 } \\
\text { C-1 } \\
\text { C-4 } \\
\text { C-1 }\end{array}$ \\
\hline $\begin{array}{l}12 \\
12 \\
12 \\
12 \\
12\end{array}$ & $\begin{array}{l}2 \\
2 \\
3 \\
3 \\
3\end{array}$ & $\begin{array}{c}84-131 \\
131-150 \\
0-30 \\
30-64 \\
64-150\end{array}$ & $\begin{array}{l}C-4 \\
C-1 \\
C-1 \\
C-4 \\
C-1\end{array}$ \\
\hline $\begin{array}{l}12 \\
12 \\
12 \\
12 \\
12\end{array}$ & $\begin{array}{l}4 \\
4 \\
4 \\
5 \\
5\end{array}$ & $\begin{array}{c}0-55 \\
55-97 \\
97-150 \\
0-63 \\
63-150\end{array}$ & $\begin{array}{l}\text { C-1 } \\
\text { C-4 } \\
\text { C-1 } \\
\text { C-1 } \\
\text { C-4 }\end{array}$ \\
\hline $\begin{array}{l}13 \\
13 \\
13 \\
13 \\
13\end{array}$ & $\begin{array}{l}1 \\
2 \\
2 \\
2 \\
2\end{array}$ & $\begin{array}{r}0-36 \\
36-45 \\
45-73 \\
73-80\end{array}$ & $\begin{array}{l}C-1 \\
C-1 \\
C-4 \\
C-1 \\
C-4\end{array}$ \\
\hline $\begin{array}{l}13 \\
13 \\
13 \\
13 \\
13\end{array}$ & $\begin{array}{l}2 \\
2 \\
2 \\
2 \\
2\end{array}$ & $\begin{array}{r}80-117 \\
117-129 \\
129-137 \\
137-146 \\
146-150\end{array}$ & $\begin{array}{l}C-1 \\
C-4 \\
C-1 \\
C-4 \\
C-1\end{array}$ \\
\hline $\begin{array}{l}13 \\
13 \\
13 \\
14 \\
14\end{array}$ & $\begin{array}{l}3 \\
3 \\
3 \\
1 \\
1\end{array}$ & $\begin{array}{c}0-12 \\
12-25 \\
25-150 \\
0-47 \\
47-51\end{array}$ & $\begin{array}{l}\text { C-4 } \\
\text { C-1 } \\
\text { C-4 } \\
\text { C-1 } \\
\text { C-4 }\end{array}$ \\
\hline
\end{tabular}

TABLE 3 - Continued

\begin{tabular}{|c|c|c|c|}
\hline Core & Section & $\begin{array}{c}\text { Interval } \\
(\mathrm{cm})\end{array}$ & Geometric Condition $\mathrm{b}$ \\
\hline \multicolumn{4}{|c|}{ Hole 416A } \\
\hline $\begin{array}{l}14 \\
14 \\
14 \\
14 \\
14\end{array}$ & $\begin{array}{l}1 \\
2 \\
2 \\
2 \\
2\end{array}$ & $\begin{array}{c}51-150 \\
0-37 \\
37-105 \\
105-132 \\
132-136\end{array}$ & $\begin{array}{l}\text { C-1 } \\
\text { C-4 } \\
\text { C-1 } \\
\text { C-4 } \\
\text { C-1 }\end{array}$ \\
\hline $\begin{array}{l}14 \\
14 \\
14 \\
14 \\
14\end{array}$ & $\begin{array}{l}2 \\
3 \\
3 \\
3 \\
3\end{array}$ & $\begin{array}{c}136-150 \\
0-32 \\
32-41 \\
41-70 \\
70-90\end{array}$ & $\begin{array}{l}\text { C-4 } \\
\text { C-4 } \\
\text { C-1 } \\
\text { C-4 } \\
\text { C-1 }\end{array}$ \\
\hline $\begin{array}{l}14 \\
14 \\
14 \\
14 \\
14\end{array}$ & $\begin{array}{l}3 \\
3 \\
3 \\
3 \\
4\end{array}$ & $\begin{array}{r}90-110 \\
110-125 \\
125-134 \\
134-150 \\
0-50\end{array}$ & $\begin{array}{l}C-4 \\
C-1 \\
C-4 \\
C-1 \\
C-1\end{array}$ \\
\hline $\begin{array}{l}14 \\
14 \\
14 \\
14 \\
14\end{array}$ & $\begin{array}{l}4 \\
4 \\
5 \\
5 \\
5\end{array}$ & $\begin{array}{c}50-55 \\
55-150 \\
0-44 \\
44-63 \\
63-80\end{array}$ & $\begin{array}{l}\text { C-3 } \\
\text { C-1 } \\
\text { C-1 } \\
\text { C-4 } \\
\text { C-1 }\end{array}$ \\
\hline $\begin{array}{l}14 \\
14 \\
14 \\
14 \\
14\end{array}$ & $\begin{array}{l}5 \\
5 \\
5 \\
5 \\
5\end{array}$ & $\begin{array}{c}80-87 \\
87-107 \\
107-110 \\
110-117 \\
117-150\end{array}$ & $\begin{array}{l}\text { C-4 } \\
\text { C-1 } \\
\text { C-4 } \\
\text { C-1 } \\
\text { C-4 }\end{array}$ \\
\hline $\begin{array}{l}15 \\
15 \\
15 \\
15 \\
15\end{array}$ & $\begin{array}{l}1 \\
1 \\
1 \\
1 \\
1\end{array}$ & $\begin{array}{r}0-107 \\
107-112 \\
112-120 \\
120-124 \\
124-150\end{array}$ & $\begin{array}{l}\text { C-1 } \\
\text { C-4 } \\
\text { C-1 } \\
\text { C-4 } \\
\text { C-1 }\end{array}$ \\
\hline $\begin{array}{l}15 \\
15 \\
15 \\
15 \\
15\end{array}$ & $\begin{array}{l}2 \\
3 \\
3 \\
3 \\
4\end{array}$ & $\begin{array}{c}0-12 \\
12-19 \\
19-150\end{array}$ & $\begin{array}{l}C-1 \\
C-1 \\
C-4 \\
C-1 \\
C-1\end{array}$ \\
\hline $\begin{array}{l}15 \\
15 \\
15 \\
15 \\
15\end{array}$ & $\begin{array}{l}5 \\
5 \\
5 \\
6 \\
6\end{array}$ & $\begin{array}{c}0-44 \\
44-70 \\
70-150 \\
0-62 \\
62-150\end{array}$ & $\begin{array}{l}\text { C-1 } \\
\text { C-4 } \\
\text { C-1 } \\
\text { C-4 } \\
\text { C-1 }\end{array}$ \\
\hline $\begin{array}{l}16 \\
16 \\
16 \\
16 \\
16\end{array}$ & $\begin{array}{l}1 \\
2 \\
2 \\
2 \\
2\end{array}$ & $\begin{array}{c}0-25 \\
25-42 \\
42-84 \\
84-110\end{array}$ & $\begin{array}{l}\text { C-1 } \\
\text { C-4 } \\
\text { C-1 } \\
\text { C-4 } \\
\text { C-1 }\end{array}$ \\
\hline $\begin{array}{l}16 \\
16 \\
16 \\
16 \\
16\end{array}$ & $\begin{array}{l}2 \\
2 \\
3 \\
3 \\
3\end{array}$ & $\begin{array}{c}110-138 \\
138-150 \\
0-8 \\
8-26 \\
26-40\end{array}$ & $\begin{array}{l}\text { C-4 } \\
\text { C-1 } \\
\text { C-1 } \\
\text { C-4 } \\
\text { C-1 }\end{array}$ \\
\hline $\begin{array}{l}16 \\
16 \\
16 \\
16 \\
16\end{array}$ & $\begin{array}{l}3 \\
3 \\
3 \\
3 \\
4\end{array}$ & $\begin{array}{c}40-85 \\
85-105 \\
105-138 \\
138-150 \\
0-47\end{array}$ & $\begin{array}{l}\text { C-4 } \\
\text { C-1 } \\
\text { C-4 } \\
\text { C-1 } \\
\text { C-1 }\end{array}$ \\
\hline $\begin{array}{l}16 \\
16 \\
16 \\
16 \\
16\end{array}$ & $\begin{array}{l}4 \\
4 \\
4 \\
5 \\
5\end{array}$ & $\begin{array}{c}47-73 \\
73-86 \\
86-150 \\
0-23 \\
23-150\end{array}$ & $\begin{array}{l}\text { C-4 } \\
\text { C-1 } \\
\text { C-4 } \\
\text { C-1 } \\
\text { C-4 }\end{array}$ \\
\hline
\end{tabular}


TABLE 3 - Continued

\begin{tabular}{|c|c|c|c|}
\hline Core & Section & $\begin{array}{l}\text { Interval } \\
(\mathrm{cm})\end{array}$ & Geometric Condition $\mathrm{b}$ \\
\hline \multicolumn{4}{|c|}{ Hole 416A } \\
\hline $\begin{array}{l}17 \\
17 \\
17 \\
17 \\
17\end{array}$ & $\begin{array}{l}1 \\
1 \\
1 \\
2 \\
3\end{array}$ & $\begin{array}{c}0-71 \\
71-137 \\
137-150 \\
0-30\end{array}$ & $\begin{array}{l}\text { C-4 } \\
\text { C-1 } \\
\text { C-4 } \\
\text { C-4 } \\
\text { C-4 }\end{array}$ \\
\hline $\begin{array}{l}17 \\
17 \\
17 \\
17 \\
17\end{array}$ & $\begin{array}{l}3 \\
3 \\
4 \\
4 \\
4\end{array}$ & $\begin{array}{c}30-75 \\
75-150 \\
0-15 \\
15-31 \\
31-150\end{array}$ & $\begin{array}{l}\text { C-1 } \\
\text { C-4 } \\
\text { C-4 } \\
\text { C-1 } \\
\text { C-4 }\end{array}$ \\
\hline $\begin{array}{l}18 \\
18 \\
18 \\
18 \\
18\end{array}$ & $\begin{array}{l}1 \\
2 \\
2 \\
2 \\
2\end{array}$ & $\begin{array}{c}0-28 \\
28-67 \\
67-73 \\
73-100\end{array}$ & $\begin{array}{l}\text { C-1 } \\
\text { C-1 } \\
\text { C-4 } \\
\text { C-1 } \\
\text { C-4 }\end{array}$ \\
\hline $\begin{array}{l}18 \\
18 \\
18 \\
18 \\
18\end{array}$ & $\begin{array}{l}2 \\
2 \\
2 \\
3 \\
3\end{array}$ & $\begin{array}{c}100-118 \\
118-126 \\
126-150 \\
0-20 \\
20-34\end{array}$ & $\begin{array}{l}\text { C-1 } \\
\text { C-4 } \\
\text { C-1 } \\
\text { C-4 } \\
\text { C-1 }\end{array}$ \\
\hline $\begin{array}{l}18 \\
18 \\
18 \\
18 \\
18\end{array}$ & $\begin{array}{l}3 \\
3 \\
4 \\
4 \\
4\end{array}$ & $\begin{array}{c}34-50 \\
50-150 \\
0-40 \\
40-90 \\
90-122\end{array}$ & $\begin{array}{l}\text { C-4 } \\
\text { C-1 } \\
\text { C-1 } \\
\text { C-4 } \\
\text { C-1 }\end{array}$ \\
\hline $\begin{array}{l}18 \\
18 \\
19 \\
19 \\
19\end{array}$ & $\begin{array}{l}4 \\
5 \\
1 \\
1 \\
1\end{array}$ & $\begin{array}{c}122-150 \\
0-46 \\
46-54 \\
54-150\end{array}$ & $\begin{array}{l}\text { C-4 } \\
\text { C-1 } \\
\text { C-1 } \\
\text { C-4 } \\
\text { C-1 }\end{array}$ \\
\hline $\begin{array}{l}19 \\
19 \\
19 \\
19 \\
19\end{array}$ & $\begin{array}{l}2 \\
2 \\
2 \\
3 \\
3\end{array}$ & $\begin{array}{c}0-74 \\
74-83 \\
83-150 \\
0-55 \\
55-76\end{array}$ & $\begin{array}{l}\text { C-4 } \\
\text { C-1 } \\
\text { C-1 } \\
\text { C-1 } \\
\text { C-4 }\end{array}$ \\
\hline $\begin{array}{l}19 \\
19 \\
19 \\
19 \\
19\end{array}$ & $\begin{array}{l}3 \\
4 \\
4 \\
4 \\
5\end{array}$ & $\begin{array}{c}76-150 \\
0-85 \\
85-107 \\
107-150 \\
0-23\end{array}$ & $\begin{array}{l}\text { C-1 } \\
\text { C-4 } \\
\text { C-1 } \\
\text { C-4 } \\
\text { C-4 }\end{array}$ \\
\hline $\begin{array}{l}19 \\
19 \\
19 \\
20 \\
20\end{array}$ & $\begin{array}{l}5 \\
5 \\
5 \\
1 \\
2\end{array}$ & $\begin{array}{l}23-43 \\
43-66 \\
66-150 \\
\\
0-11\end{array}$ & $\begin{array}{l}\text { C-1 } \\
\text { C-4 } \\
\text { C-1 } \\
\text { C-1 } \\
\text { C-4 }\end{array}$ \\
\hline $\begin{array}{l}20 \\
20 \\
20 \\
20 \\
21\end{array}$ & $\begin{array}{l}2 \\
2 \\
2 \\
3 \\
1\end{array}$ & $\begin{array}{r}11-125 \\
125-135 \\
135-150\end{array}$ & $\begin{array}{l}\text { C-1 } \\
\text { C-4 } \\
\text { C-1 } \\
\text { C-4 }\end{array}$ \\
\hline $\begin{array}{l}21 \\
21 \\
21 \\
21 \\
21\end{array}$ & $\begin{array}{l}2 \\
3 \\
3 \\
3 \\
3\end{array}$ & $\begin{array}{r}0-22 \\
22-28 \\
28-33 \\
33-36\end{array}$ & $\begin{array}{l}\text { C-4 } \\
\text { C-1 } \\
\text { C-4 } \\
\text { C-1 }\end{array}$ \\
\hline $\begin{array}{l}21 \\
21 \\
21 \\
21 \\
21\end{array}$ & $\begin{array}{l}3 \\
3 \\
3 \\
3 \\
3\end{array}$ & $\begin{array}{c}36-69 \\
69-92 \\
92-99 \\
99-130 \\
130-150\end{array}$ & $\begin{array}{l}\text { C-4 } \\
\text { C-1 } \\
\text { C-4 } \\
\text { C-1 } \\
\text { C-4 }\end{array}$ \\
\hline
\end{tabular}

TABLE 3 - Continued

\begin{tabular}{|c|c|c|c|}
\hline Core & Section & $\begin{array}{c}\text { Interval } \\
(\mathrm{cm})\end{array}$ & Geometric Condition $\mathrm{b}$ \\
\hline \multicolumn{4}{|c|}{ Hole 416A } \\
\hline $\begin{array}{l}21 \\
21 \\
21 \\
21 \\
21\end{array}$ & $\begin{array}{l}4 \\
4 \\
4 \\
4 \\
4\end{array}$ & $\begin{array}{l}0-36 \\
36-40 \\
40-55 \\
55-70 \\
70-150\end{array}$ & $\begin{array}{l}\text { C-4 } \\
\text { C-1 } \\
\text { C-4 } \\
\text { C-1 } \\
\text { C-4 }\end{array}$ \\
\hline $\begin{array}{l}21 \\
21 \\
22 \\
22 \\
22\end{array}$ & $\begin{array}{l}5 \\
6 \\
1 \\
1 \\
1\end{array}$ & $\begin{array}{r}0-30 \\
30-35 \\
35-58\end{array}$ & $\begin{array}{l}\text { C-1 } \\
\text { C-4 } \\
\text { C-4 } \\
\text { C-1 } \\
\text { C-4 }\end{array}$ \\
\hline $\begin{array}{l}22 \\
22 \\
22 \\
22 \\
22\end{array}$ & $\begin{array}{l}1 \\
2 \\
2 \\
2 \\
2\end{array}$ & $\begin{array}{c}58-150 \\
0-26 \\
26-55 \\
55-67 \\
67-73\end{array}$ & $\begin{array}{l}\text { C-1 } \\
\text { C-1 } \\
\text { C-4 } \\
\text { C-1 } \\
\text { C-4 }\end{array}$ \\
\hline $\begin{array}{l}22 \\
22 \\
22 \\
22 \\
22 \\
22\end{array}$ & $\begin{array}{l}2 \\
2 \\
2 \\
3 \\
3 \\
3\end{array}$ & $\begin{array}{c}73-100 \\
100-120 \\
120-150 \\
0-45 \\
45-78 \\
78-94\end{array}$ & $\begin{array}{l}\text { C-1 } \\
\text { C-4 } \\
\text { C-1 } \\
\text { C-1 } \\
\text { C-4 } \\
\text { C-1 }\end{array}$ \\
\hline $\begin{array}{l}22 \\
22 \\
22 \\
22 \\
22\end{array}$ & $\begin{array}{l}3 \\
3 \\
3 \\
4 \\
4\end{array}$ & $\begin{array}{c}94-100 \\
100-111 \\
111-150 \\
0-23 \\
23-57\end{array}$ & $\begin{array}{l}\text { C-4 } \\
\text { C-1 } \\
\text { C-4 } \\
\text { C-4 } \\
\text { C-1 }\end{array}$ \\
\hline $\begin{array}{l}22 \\
22 \\
22 \\
22 \\
22\end{array}$ & $\begin{array}{l}4 \\
4 \\
4 \\
4 \\
5\end{array}$ & $\begin{array}{c}57-62 \\
62-81 \\
81-115 \\
115-150\end{array}$ & $\begin{array}{l}\text { C-4 } \\
\text { C-1 } \\
\text { C-4 } \\
\text { C-1 } \\
\text { C-4 }\end{array}$ \\
\hline $\begin{array}{l}23 \\
23 \\
23 \\
23 \\
23\end{array}$ & $\begin{array}{l}1 \\
2 \\
2 \\
2 \\
3\end{array}$ & $\begin{array}{c}0-59 \\
59-67 \\
67-150\end{array}$ & $\begin{array}{l}\text { C-1 } \\
\text { C-1 } \\
\text { C-4 } \\
\text { C-1 } \\
\text { C-1 }\end{array}$ \\
\hline $\begin{array}{l}23 \\
23 \\
23 \\
23 \\
23\end{array}$ & $\begin{array}{l}4 \\
5 \\
5 \\
5 \\
5\end{array}$ & $\begin{array}{r}0-75 \\
75-80 \\
80-85 \\
85-92\end{array}$ & $\begin{array}{l}\text { C-1 } \\
\text { C-1 } \\
\text { C-4 } \\
\text { C-1 } \\
\text { C-4 }\end{array}$ \\
\hline $\begin{array}{l}23 \\
23 \\
24 \\
24 \\
24\end{array}$ & $\begin{array}{l}5 \\
5 \\
1 \\
1 \\
1\end{array}$ & $\begin{array}{c}92-95 \\
95-150 \\
0-73 \\
73-119 \\
119-150\end{array}$ & $\begin{array}{l}C-1 \\
C-4 \\
C-1 \\
C-4 \\
C-1\end{array}$ \\
\hline $\begin{array}{l}24 \\
24 \\
24 \\
24 \\
24\end{array}$ & $\begin{array}{l}2 \\
2 \\
2 \\
3 \\
3\end{array}$ & $\begin{array}{c}0-30 \\
30-60 \\
60-150 \\
0-25 \\
25-27\end{array}$ & $\begin{array}{l}C-1 \\
C-4 \\
C-1 \\
C-4 \\
C-1\end{array}$ \\
\hline $\begin{array}{l}24 \\
24 \\
25 \\
25 \\
25\end{array}$ & $\begin{array}{l}3 \\
3 \\
1 \\
1 \\
1\end{array}$ & $\begin{array}{c}27-70 \\
70-150 \\
0-75 \\
75-92 \\
92-150\end{array}$ & $\begin{array}{l}\text { C-4 } \\
\text { C-1 } \\
\text { C-1 } \\
\text { C-4 } \\
\text { C-1 }\end{array}$ \\
\hline $\begin{array}{l}25 \\
25 \\
25 \\
25 \\
25\end{array}$ & $\begin{array}{l}2 \\
3 \\
3 \\
3 \\
4\end{array}$ & $\begin{array}{c}0-100 \\
100-130 \\
130-150 \\
0-39\end{array}$ & $\begin{array}{l}\text { C-1 } \\
\text { C-1 } \\
\text { C-4 } \\
\text { C-1 } \\
\text { C-1 }\end{array}$ \\
\hline
\end{tabular}


TABLE 3 - Continued

\begin{tabular}{|c|c|c|c|}
\hline Core & Section & $\begin{array}{l}\text { Interval } \\
(\mathrm{cm})\end{array}$ & Geometric Condition ${ }^{b}$ \\
\hline \multicolumn{4}{|c|}{ Hole 416A } \\
\hline $\begin{array}{l}25 \\
25 \\
25 \\
26 \\
27\end{array}$ & $\begin{array}{l}4 \\
4 \\
5 \\
1\end{array}$ & $\begin{array}{l}39-50 \\
50-150\end{array}$ & $\begin{array}{l}\text { C-4 } \\
C-1 \\
C-1 \\
\text { C-1 } \\
\text { C-1 }\end{array}$ \\
\hline $\begin{array}{l}27 \\
27 \\
27 \\
27 \\
27\end{array}$ & $\begin{array}{c}2 \\
2 \\
2 \\
3 \text { through } 4 \\
5\end{array}$ & $\begin{array}{r}0-100 \\
100-125 \\
125-150 \\
0-65\end{array}$ & $\begin{array}{l}C-1 \\
C-4 \\
C-1 \\
C-1 \\
C-1\end{array}$ \\
\hline $\begin{array}{l}27 \\
27 \\
27 \\
28 \\
28\end{array}$ & $\begin{array}{c}5 \\
5 \\
6 \\
1 \text { through } 3 \\
4\end{array}$ & $\begin{array}{r}65-110 \\
110-150 \\
0-35\end{array}$ & $\begin{array}{l}C-4 \\
C-1 \\
C-1 \\
C-1 \\
C-1\end{array}$ \\
\hline $\begin{array}{l}28 \\
28 \\
28 \\
28 \\
28\end{array}$ & $\begin{array}{l}4 \\
4 \\
5 \\
6 \\
6\end{array}$ & $\begin{array}{l}35-60 \\
60-150 \\
0-80 \\
80-85\end{array}$ & $\begin{array}{l}C-3 \\
C-1 \\
C-1 \\
C-1 \\
C-4\end{array}$ \\
\hline $\begin{array}{l}28 \\
29 \\
30 \\
30 \\
30\end{array}$ & $\begin{array}{c}6 \\
1 \text { through } 5 \\
6 \\
6\end{array}$ & $\begin{array}{r}85-150 \\
\\
0-47 \\
47-85\end{array}$ & $\begin{array}{l}\text { C-1 } \\
\text { C-1 } \\
\text { C-1 } \\
\text { C-4 } \\
\text { C-1 }\end{array}$ \\
\hline $\begin{array}{l}30 \\
31 \\
31 \\
31 \\
31\end{array}$ & $\begin{array}{l}6 \\
1 \\
2 \\
2 \\
2\end{array}$ & $\begin{array}{r}85-150 \\
0-104 \\
104-118 \\
118-150\end{array}$ & $\begin{array}{l}C-4 \\
C-1 \\
C-1 \\
C-4 \\
C-1\end{array}$ \\
\hline $\begin{array}{l}31 \\
31 \\
31 \\
32 \\
33\end{array}$ & $\begin{array}{c}3 \text { through } 5 \\
6 \\
6\end{array}$ & $\begin{array}{c}0-82 \\
82-150\end{array}$ & $\begin{array}{l}\text { C-1 } \\
\text { C-4 } \\
\text { C-1 } \\
\text { C-1 } \\
\text { C-4 }\end{array}$ \\
\hline $\begin{array}{l}34 \\
34 \\
34 \\
34 \\
34\end{array}$ & $\begin{array}{l}1 \\
2 \\
2 \\
2 \\
2\end{array}$ & $\begin{array}{c}0-20 \\
20-30 \\
30-112 \\
112-125\end{array}$ & $\begin{array}{l}C-1 \\
C-1 \\
C-4 \\
C-1 \\
C-4\end{array}$ \\
\hline $\begin{array}{l}34 \\
34 \\
34 \\
34 \\
34\end{array}$ & $\begin{array}{l}2 \\
3 \\
3 \\
3 \\
4\end{array}$ & $\begin{array}{c}125-150 \\
0-83 \\
83-102 \\
102-150\end{array}$ & $\begin{array}{l}C-1 \\
C-1 \\
C-4 \\
C-1 \\
C-1\end{array}$ \\
\hline $\begin{array}{c}35 \\
35 \\
35 \\
36-38 \\
40\end{array}$ & $\begin{array}{c}1 ; 2 \\
3 \\
3 \\
1 \text { through } 5\end{array}$ & $\begin{array}{r}0-104 \\
104-150\end{array}$ & $\begin{array}{l}C-1 \\
C-1 \\
C-4 \\
C-1 \\
C-1\end{array}$ \\
\hline $\begin{array}{l}40 \\
40 \\
40 \\
41 \\
41\end{array}$ & $\begin{array}{l}6 \\
6 \\
6 \\
1 \\
1\end{array}$ & $\begin{array}{c}0-40 \\
40-92 \\
92-150 \\
0-30 \\
30-37\end{array}$ & $\begin{array}{l}C-1 \\
C-4 \\
C-1 \\
C-1 \\
C-4\end{array}$ \\
\hline $\begin{array}{l}41 \\
41 \\
41 \\
41 \\
41\end{array}$ & $\begin{array}{c}1 \\
2 \text { through } 4 \\
5 \\
5 \\
5\end{array}$ & $\begin{array}{c}37-150 \\
\\
0-45 \\
45-70 \\
70-150\end{array}$ & $\begin{array}{l}\text { C-1 } \\
\text { C-1 } \\
\text { C-1 } \\
\text { C-4 } \\
\text { C-1 }\end{array}$ \\
\hline
\end{tabular}

TABLE 3 - Continued

\begin{tabular}{cccc}
\hline Core & Section & $\begin{array}{c}\text { Interval } \\
(\mathrm{cm})\end{array}$ & Geometric Conditionb \\
\hline Hole $416 \mathrm{~A}$ & & & \\
42 & $1 ; 2$ & & $\mathrm{C}-1$ \\
42 & 3 & $0-16$ & $\mathrm{C}-4$ \\
42 & 3 & $16-150$ & $\mathrm{C}-1$ \\
43 & & & \\
44 & 1 & & $\mathrm{C}-1$ \\
44 & 2 & $0-50$ & $\mathrm{C}-1$ \\
44 & 2 & $50-63$ & $\mathrm{C}-4$ \\
44 & 2 & $63-150$ & $\mathrm{C}-1$ \\
45 & 1 & $0-100$ & $\mathrm{C}-1$ \\
45 & 1 & $100-150$ & $\mathrm{C}-4$ \\
45 & $2 ; 3$ & & $\mathrm{C}-1$ \\
$46-48$ & & & $\mathrm{C}-1$ \\
49 & 1 & & $\mathrm{C}-1$ \\
49 & 2 & $0-28$ & $\mathrm{C}-1$ \\
49 & 2 & $28-44$ & $\mathrm{C}-4$ \\
49 & 2 & $44-150$ & $\mathrm{C}-1$ \\
$50-52$ & & & $\mathrm{C}-1$ \\
53 & 1 & $0-112$ & $\mathrm{C}-1$ \\
53 & 1 & $112-150$ & $\mathrm{C}-4$ \\
53 & $2 ; 3$ & & C-4 \\
54 & 1 & & A \\
54 & 2 & & $\mathrm{C}-1$ \\
55 & & & $\mathrm{C}-1$ \\
57 & & &
\end{tabular}

${ }^{a}$ Because the type of material surrounding the cores at Hole 415 through core $416 \mathrm{~A}-22$ was not recorded consistently aboard ship (owing to the many people involved), the information may contain errors. If any density data appear anomalous, a future investigator may wish to recalculate densities by using different "geometric conditions" as models. The conditions listed here were reconstructed from photographs and used in the computer program.

$\mathrm{b}_{\text {Code } \mathrm{A}}=$ Soft Sediment in Liner: Soft sediment completely filled the core liner and no diameter adjustments were necessary.

Code $\mathrm{C}=$ Hard Rock in Liner: Code notes that the rock is surrounded by (with "corrected density"):

Code $\mathrm{C}-1=$ air, $0.0 \mathrm{~g} / \mathrm{cm}^{3}$.

Code $\mathrm{C}-2=$ water, $1.1 \mathrm{~g} / \mathrm{cm}^{3}$.

Code $\mathrm{C}-3$ = sediment, $1.6 \mathrm{~g} / \mathrm{cm}^{3}$.

Code $\mathrm{C}-4$ = breccia, $1.8 \mathrm{~g} / \mathrm{cm}^{3}$.

If rock is surrounded by water or air, then the diameter is offset from the gamma-ray beam axis $3.305 \mathrm{~cm}$ minus radius of the rock.

stant value of 0.1024 (the average $\mu_{\mathrm{a}}$ in Table 5) for Hole 416A Cores 17 through 57.

For each GRAPE 2-minute count, we measured the sample thickness, the gamma count through the sample, and a gamma count in air for each sample. We used these values in Equation 24 (Boyce, 1976) to calculate the Evans (1965) corrected wet-bulk density $\left(\varrho_{\mathrm{bc}}\right)$, using a constant $\varrho_{\mathrm{a}}$ of $0.1028 \mathrm{~cm}^{2} / \mathrm{g}$ for samples from Site 415 and Hole $416 \mathrm{~A}$ through Core 16, and a constant $\varrho_{\mathrm{a}}$ of 0.1024 for Site $416 \mathrm{~A}$, Cores 17 through 57 . We then took this $\varrho_{\mathrm{bc}}$, derived from Equation 24, and placed it into Equation 21 of Boyce (1976) to calculate "true" wet-bulk density. The following numerical data were used in Equation 21: (1) grain density $\left(\varrho_{\mathrm{g}}\right)=2.7 \mathrm{~g} / \mathrm{cm}^{3}$; 
TABLE 4

Apparent Quartz Attenuation Coefficient Determined from the $6.61^{\mathrm{a}}$ and $2.54^{\mathrm{b}} \mathrm{cm}$ Aluminum Standards Processed from Site 415 through Hole 416A, Core $16^{\mathrm{c}}$

\begin{tabular}{lccc}
\hline $\begin{array}{c}\text { Aluminum d } \\
2.54 \mathrm{~cm}\end{array}$ & $\begin{array}{c}\text { Aluminum } \\
6.61 \mathrm{~cm}\end{array}$ & Between & \\
$\left(\mathrm{cm}^{2} / \mathrm{g}\right)$ & $\left(\mathrm{cm}^{2} / \mathrm{g}\right)$ & Cores & Date \\
\hline
\end{tabular}

Hole 415

\begin{tabular}{|c|c|c|c|}
\hline \\
\hline & 0.1030 & $0-1$ & 13 September \\
\hline & 0.1036 & $0-1$ & 13 September \\
\hline & 0.1029 & $0-1$ & 13 September \\
\hline & 0.1031 & $0-1$ & 13 September \\
\hline & 0.1031 & $0-1$ & 13 September \\
\hline & 0.1031 & $0-1$ & 13 September \\
\hline & 0.1030 & $0-1$ & 13 September \\
\hline & 0.1027 & $0-1$ & 13 September \\
\hline & 0.1032 & $0-1$ & 13 September \\
\hline & 0.1029 & $0-1$ & 13 September \\
\hline & 0.1027 & $0-1$ & 13 September \\
\hline & 0.1031 & $0-1$ & 13 September \\
\hline 0.1023 & & $0-1$ & 13 September \\
\hline 0.1030 & & $0-1$ & 13 September \\
\hline 0.1017 & & $0-1$ & 13 September \\
\hline 0.1012 & & $0-1$ & 13 September \\
\hline 0.1017 & & $0-1$ & 13 September \\
\hline 0.1024 & & $0-1$ & 13 September \\
\hline 0.1016 & & $0-1$ & 13 September \\
\hline 0.1026 & & $0-1$ & 13 September \\
\hline 0.1021 & & $0-1$ & 13 September \\
\hline \multirow[t]{4}{*}{0.1024} & & $0-1$ & 13 September \\
\hline & 0.1026 & $0-1$ & 13 September \\
\hline & 0.1026 & $0-1$ & 13 September \\
\hline & 0.1027 & $0-1$ & 13 September \\
\hline 0.1032 & 0.1027 & $0-1$ & 15 September \\
\hline 0.1018 & 0.1023 & $4-5$ & 16 September \\
\hline \multicolumn{4}{|c|}{ Hole 415A } \\
\hline 0.1029 & 0.1034 & $4-5$ & 23 September \\
\hline \multicolumn{4}{|c|}{ Hole 416A } \\
\hline 0.1041 & 0.1033 & $3-4$ & 3 October \\
\hline 0.1031 & 0.1031 & $5-6$ & 6 October \\
\hline 0.1042 & 0.1022 & 7 & 6 October \\
\hline 0.1033 & 0.1027 & $7-8$ & 11 October \\
\hline 0.1035 & 0.1032 & $13-14$ & 11 October \\
\hline 0.1028 & 0.1027 & $15-16$ & 11 October \\
\hline \multicolumn{4}{|c|}{$\begin{array}{l}\text { a Al } 6.61 \mathrm{~cm} \text { average }=0.1029 \mathrm{~cm}^{2} / \mathrm{g} . \\
\mathrm{b}_{\mathrm{Al}} 2.54 \mathrm{~cm} \text { average }=0.1026 \mathrm{~cm}^{2} / \mathrm{g} . \\
\mathrm{c}_{\text {The average } \mu \text { which is used in calculation of GRAPE }} \\
\text { special } 2 \text {-minute count wet-bulk density }= \\
\frac{0.1026 \mathrm{~cm}^{2} / \mathrm{g}+0.1029 \mathrm{~cm}^{2} / \mathrm{g}}{2}=0.1028 \mathrm{~cm}^{2} / \mathrm{g} \text { for }\end{array}$} \\
\hline \multicolumn{4}{|c|}{$\begin{array}{l}\text { Site } 415 \text { through Hole } A \text {, Core } 16 . \\
\mathrm{d}_{\text {Apparent quartz attenuation coefficient }}=\frac{\ln \mathrm{I}_{\mathrm{O}} / \mathrm{I}}{\mathrm{d}(2.60 \mathrm{~g} / \mathrm{cc})} \\
\text { where } \mathrm{I}_{\mathrm{O}}=\text { gamma count through air, } \mathrm{I}=\text { gamma count } \\
\text { through aluminum standard, } \mathrm{d}=\text { thickness of standard } \\
(6.61 \mathrm{~cm} \text { or } 2.54 \mathrm{~cm}) .\end{array}$} \\
\hline
\end{tabular}

(2) corrected grain density $\left(\varrho_{\mathrm{gc}}\right)=2.7 \mathrm{~g} / \mathrm{cm}^{3}$; (3) fluid density $\left(\varrho_{\mathrm{f}}\right)=1.025 \mathrm{~g} / \mathrm{cm}^{3}$; and (4) corrected fluid density $\left(\varrho_{\mathrm{fc}}\right)=1.128 \mathrm{~g} / \mathrm{cm}^{3}$. This "true" wet-bulk density derived from Equation 21 is the value published in the Leg 50 volume. See Boyce (1976) for discussions of errors and assumptions relating to these formulas.
TABLE 5

Apparent Attenuation Coefficients Determined from the $6.61^{\mathrm{a}} \mathrm{cm}$ and $2.54^{\mathrm{b}} \mathrm{cm}$ Aluminum Standards Processed during Hole 416A, Cores $17-57^{\mathrm{C}}$

\begin{tabular}{|c|c|c|c|c|}
\hline $\begin{array}{l}\text { Aluminumd } \\
2.54 \mathrm{~cm} \\
\left(\mathrm{~cm}^{2} / \mathrm{g}\right)\end{array}$ & $\begin{array}{l}\text { Aluminumd } \\
6.61 \mathrm{~cm} \\
\left(\mathrm{~cm}^{2} / \mathrm{g}\right)\end{array}$ & Hole & $\begin{array}{l}\text { Between } \\
\text { Cores }\end{array}$ & Date \\
\hline 0.1019 & 0.1027 & $416 \mathrm{~A}$ & $17-18$ & 13 October \\
\hline 0.1009 & 0.1024 & $416 \mathrm{~A}$ & $18-19$ & 13 October \\
\hline 0.1022 & 0.1027 & $416 \mathrm{~A}$ & $19-20$ & 13 October \\
\hline 0.1010 & 0.1025 & $416 \mathrm{~A}$ & $20-21$ & 13 October \\
\hline 0.1038 & 0.1029 & $416 \mathrm{~A}$ & $21-22$ & 13 October \\
\hline 0.1035 & 0.1025 & $416 \mathrm{~A}$ & $22-23$ & 14 October \\
\hline 0.1027 & 0.1040 & $416 \mathrm{~A}$ & $22-23$ & 14 October \\
\hline 0.1028 & $\begin{array}{l}\text { air count } \\
\text { too high }\end{array}$ & & & 14 October \\
\hline 0.1022 & 0.1026 & $416 \mathrm{~A}$ & $24-25$ & 14 October \\
\hline 0.1006 & 0.1022 & $416 \mathrm{~A}$ & $25-26$ & 15 October \\
\hline 0.1026 & 0.1023 & $416 \mathrm{~A}$ & $28-29$ & 15 October \\
\hline 0.1025 & 0.1022 & $416 \mathrm{~A}$ & 29-31 & 15 October \\
\hline 0.1022 & 0.1028 & $416 \mathrm{~A}$ & $33-34$ & 18 October \\
\hline 0.1019 & 0.1025 & $416 \mathrm{~A}$ & $36-37$ & 19 October \\
\hline 0.1023 & 0.1026 & $416 \mathrm{~A}$ & $37-38$ & 19 October \\
\hline 0.1019 & 0.1021 & $416 \mathrm{~A}$ & $37-38$ & 19 October \\
\hline 0.1027 & 0.1027 & $416 \mathrm{~A}$ & $37-38$ & 19 October \\
\hline 0.1032 & 0.1027 & $416 \mathrm{~A}$ & $37-38$ & 19 October \\
\hline 0.1030 & 0.1028 & $416 \mathrm{~A}$ & $37-38$ & 19 October \\
\hline 0.1027 & 0.1023 & $416 \mathrm{~A}$ & $39-40$ & 20 October \\
\hline 0.1019 & 0.1024 & $416 \mathrm{~A}$ & $40-41$ & 21 October \\
\hline 0.1026 & 0.1023 & $416 \mathrm{~A}$ & $43-44$ & 21 October \\
\hline 0.1010 & 0.1027 & $416 \mathrm{~A}$ & $44-45$ & 21 October \\
\hline 0.1030 & 0.1024 & $416 \mathrm{~A}$ & $45-46$ & 22 October \\
\hline 0.0999 & 0.1026 & $416 \mathrm{~A}$ & $48-49$ & 22 October \\
\hline 0.1013 & 0.1021 & $416 \mathrm{~A}$ & $49-50$ & 23 October \\
\hline 0.1023 & 0.1031 & $416 \mathrm{~A}$ & $51-52$ & 23 October \\
\hline 0.1019 & 0.1024 & $416 \mathrm{~A}$ & $52-53$ & 23 October \\
\hline 0.1019 & 0.1024 & $416 \mathrm{~A}$ & $56-57$ & 1 November \\
\hline
\end{tabular}

${ }^{\mathrm{a}} \mathrm{Al} 6.61 \mathrm{~cm}$ average $=0.1026$.

$\mathrm{b}_{\mathrm{Al}} 2.54 \mathrm{~cm}$ average $=0.1022$.

The average $\mu_{\mathrm{a}}$ which is used in the calculation of GRAPE special 2-minute count wet bulk density $=$ $\frac{0.1022 \mathrm{~cm}^{2} / \mathrm{g}+0.1026 \mathrm{~cm}^{2} / \mathrm{g}}{2}=0.1024 \mathrm{~cm}^{2} / \mathrm{g}$.

$\mathrm{d}_{\text {Apparent quartz attenuation coefficient }}=\frac{\ln \mathrm{I}_{\mathrm{O}} / \mathrm{I}}{\mathrm{d}\left(2.60 \mathrm{~g} / \mathrm{cm}^{3}\right)}$ where $\mathrm{I}_{\mathrm{O}}=$ gamma count through air, $\mathrm{I}=$ gamma count through aluminum standard, $\mathrm{d}=$ thickness of standard $(2.54 \mathrm{~cm}$ or 6.61 $\mathrm{cm})$.

One difference in the GRAPE calculations and gravimetric calculations is that the GRAPE assumes that the sediments and rocks have a 35 per mill interstitial-water salinity. All other gravimetric wet-water-content calculations and porosity calculations assume a salinity of 45 per mill. Because of the assumptions and errors involved in the GRAPE data, this 10 per mill difference is insignificant. For simplicity, we have used constant salt corrections throughout. The Leg 50 interstitial-water salinities ranged widely, between 35 and 60 per mill, and future investigators may wish to adjust the data for intervals of varying interstitial-water salinities.

The Boyce (1976) publication on the GRAPE methods had some typographical errors:

1. Page 940, Figure 1.

The density axis should read as follows: 
Upper axis:

GAMMA-RAY “CORRECTED”' WET-BULK DENSITY

(GAMMA-RAY “CORRECTED” GRAIN DENSITY@0\% POROSITY)

(GAMMA-RAY “CORRECTED” FLUID DENSITY@100\% POROSITY)

Lower axis:

\section{TRUE WET-BULK DENSITY \\ (TRUE GRAIN DENSITY@0\% POROSITY) \\ (TRUE FLUID DENSITY @100\% POROSI- TY)}

Also in Figure 1, "1.1.128 g/cc" should read " 1.128 $\mathrm{g} / \mathrm{cc}$ "

2. Page 943. Footnote number two: "improver" should read "improper."

3. Page 944. Figure 8, caption.

Delete from lines 9, 10,11, "the $6.61 \mathrm{~cm}$ aluminum averaged apparent quartz mass attenuation coefficient $\left(0.10915 \mathrm{~cm}^{2} / \mathrm{g}\right)$ and"

4. Page 945 . First column, 19th line, delete " 2.70 " and insert " 2.71 ."

5. Page 947 . Second column, lines 20 and 21.

Put parentheses around (for cores which were in or out of liner; and surrounded with air, water, or slurry), and delete the comma after slurry.

6. Page 955. Appendix E. Second paragraph, 12th line. Change: " $\mathrm{m}_{\mathrm{a}}$ count $\mathrm{I}_{2.54}$, and the sample gamma count $\mathrm{I}_{\mathrm{o}}$, respectively, through",

To: " $\mathrm{m}_{\mathrm{a}}$ count $\mathrm{I}_{2.54}$, and the sample gamma count $\mathrm{I}_{\mathrm{s}}$, respectively, through"

\section{POROSITY}

Porosity values published with laboratory velocity data in the site chapters (this volume) are calculated from the product of 2-minute GRAPE wet-bulk density and salt-corrected $(45 \%)$ wet-water content and divided by $1.032 \mathrm{~g} / \mathrm{cm}^{3}$ density of interstitial water:

Porosity $(\%)=$

$100 \times$ salt-corrected wet-water content $) \times($ GRAPE 2 -Minute wet-bulk density)

$$
1.032 \mathrm{~g} / \mathrm{cm}^{3}
$$

The density of interstitial-water and salt-corrected wetwater assumes an interstitial-water salinity of 45 per mill (actual range during Leg 50 was 35 to $60 \%$ ). This value is used as a constant for simplicity, and future investigators may wish to use other constants. The 1.032 $\mathrm{g} / \mathrm{cm}^{3}$ density of the sea water $\left(45 \%\right.$ @ $\left.21^{\circ} \mathrm{C}\right)$ is obtained from a linear extrapolation from a density of $1.0245 \mathrm{~g} / \mathrm{cm}^{3}$ at $35.000 \%\left(21^{\circ} \mathrm{C}\right)$ and $1.0283 \mathrm{~g} / \mathrm{cm}^{3}$ at $39.99 \%$ ( $\left(21^{\circ} \mathrm{C}\right)$. The data are from "Tables for Rapid Computation of Density and Electrical Conductivity of Sea Water," U.S. Naval Hydrographic Office, Washington, D.C., Special Publication (11 May 1956, 1954 reprint).

\section{ACOUSTIC IMPEDANCE}

The acoustic impedance, in units of $\left(\mathrm{g} \times 10^{5}\right) /\left(\mathrm{cm}^{2}-\mathrm{s}\right)$, is calculated as the product of the 2-minute GRAPE wet-bulk density and vertical sound velocity.

\section{SOUND VELOCITY}

Compressional-sound velocity $(\mathrm{km} / \mathrm{s})$, at $400 \mathrm{kHz}$, through water-saturated sediments and sedimentary rock was measured with a Hamilton Frame Velocimeter which is accurate to \pm 2 per cent. The basic equipment and technique are described in Boyce (1973) and except for calibration procedures with corresponding data and sampling techniques are not described here.

\section{Oscilloscope Calibration and Velocity Correction Factors}

One oscilloscope was used during Leg 50, the DSDP Tektronix 561A. The correction factors listed in Table 6 were used to calculate the sound-velocity data for all holes drilled during Leg 50. The sound-velocity correction factors were calculated by averaging numerous velocity measurements on the lucite, brass, and aluminum semistandards, assuming that the true velocities are the Schreiber sound velocities listed in Table 7 (Boyce, 1973, 1976). Distilled water, for which acoustic velocities at given temperatures are known, was also used as a standard. Apparent-velocity measurements were averaged for each semistandard for a given $\mu \mathrm{s} / \mathrm{cm}$ setting on the oscilloscope (Tables 8 through 11). Deviations of the averages of apparent velocities from the true velocities of the semistandards, measured on the velocimeter, were used to calculate a set of correction factors $(\mathrm{K})$ for

TABLE 6

Correction Factors (K) for the DSDP Tektronics 561A Oscilloscope

\begin{tabular}{cl}
\hline $\begin{array}{c}\text { Scope Setting } \\
(\mu \mathrm{s} / \mathrm{cm})\end{array}$ & $\mathrm{K}$ \\
\hline 1.0 & Apparent velocity $\times 1.02380=$ true velocity \\
2.0 & Apparent velocity $\times 1.03779=$ true velocity \\
5.0 & Apparent velocity $\times 1.00601=$ true velocity \\
\hline
\end{tabular}

TABLE 7

Predetermined Sound Velocities of Lucite, Brass, and Aluminum Semi-Standards, as Listed in Boyce (1973)

\begin{tabular}{llll}
\hline & \multicolumn{1}{c}{ Lucite } & \multicolumn{1}{c}{ Brass } & \multicolumn{1}{c}{ Aluminum } \\
\hline Boyce (1973) & $2.741 \mathrm{~km} / \mathrm{s}$ & $4.506 \mathrm{~km} / \mathrm{s}$ & $6.293 \mathrm{~km} / \mathrm{s}$ \\
& $( \pm 0.84 \%)$ & $( \pm 0.45 \%)$ & $( \pm 1.29 \%)$ \\
Schreiber $^{\mathrm{a}}$ & $2.745 \mathrm{~km} / \mathrm{s}$ & $4.529 \mathrm{~km} / \mathrm{s}$ & $6.295 \mathrm{~km} / \mathrm{s}$ \\
& $( \pm 0.006 \mathrm{~km} / \mathrm{s})$ & $( \pm 0.004 \mathrm{~km} / \mathrm{s}$ & $( \pm 0.008 \mathrm{~km} / \mathrm{s})$ \\
\hline
\end{tabular}

${ }^{\mathrm{a}}$ Schreiber used the modified pulse transmission method (Mottaboni and Schreiber, 1967). Lamont-Doherty Geological Observatory, personal communication, 1971. 
TABLE 8

Uncorrected Velocities $(\mathrm{km} / \mathrm{s})$ through Aluminum Sonic Semi-Standards

\begin{tabular}{|c|c|c|c|c|c|c|}
\hline \multirow{2}{*}{$\begin{array}{l}\text { Scope Settinga } \\
\text { Semi-Standard } \\
\text { Thickness }\end{array}$} & \multicolumn{2}{|c|}{$1.0 \mu \mathrm{s} / \mathrm{cm}$} & \multicolumn{2}{|c|}{$2.0 \mu \mathrm{s} / \mathrm{cm}$} & \multicolumn{2}{|c|}{$5.0 \mu \mathrm{s} / \mathrm{cm}$} \\
\hline & $2.54 \mathrm{~cm}$ & $5.00 \mathrm{~cm}$ & $2.54 \mathrm{~cm}$ & $5.00 \mathrm{~cm}$ & $2.54 \mathrm{~cm}$ & $5.00 \mathrm{~cm}$ \\
\hline & $\begin{array}{c}\text { (Fair use of } \\
\text { centimeter delay) }\end{array}$ & $\begin{array}{c}\text { (Good use of } \\
\text { centimeter delay) }\end{array}$ & $\begin{array}{c}\text { (Poor use of } \\
\text { centimeter delay) }\end{array}$ & $\begin{array}{c}\text { (Fair use of } \\
\text { centimeter delay) }\end{array}$ & & $\begin{array}{c}\text { (Poor use of } \\
\text { centimeter delay) }\end{array}$ \\
\hline & $\begin{array}{l}6.084 \\
6.040 \\
6.040 \\
6.076 \\
6.052 \\
6.005 \\
6.101 \\
6.064 \\
6.108 \\
6.033\end{array}$ & $\begin{array}{l}6.194 \\
6.176 \\
6.157 \\
6.165 \\
6.163 \\
6.121 \\
6.165 \\
6.157 \\
6.166 \\
6.150\end{array}$ & $\begin{array}{l}6.057 \\
6.052 \\
5.875 \\
6.026 \\
5.889 \\
5.986 \\
5.981 \\
5.933 \\
5.956 \\
5.928\end{array}$ & $\begin{array}{l}6.133 \\
6.055 \\
6.085 \\
6.092 \\
6.057 \\
6.024 \\
6.072 \\
6.084 \\
6.136 \\
6.100\end{array}$ & & $\begin{array}{l}6.410 \\
6.356 \\
6.199 \\
6.274 \\
6.437 \\
6.204 \\
6.382 \\
6.320 \\
6.254 \\
6.335\end{array}$ \\
\hline Average & 6.060 & 6.161 & 5.968 & 6.084 & & 6.317 \\
\hline Range & $\begin{array}{l}+0.8 \% \\
-0.9 \%\end{array}$ & $\begin{array}{l}+0.5 \% \\
-0.7 \%\end{array}$ & $\begin{array}{l}+1.6 \% \\
-1.6 \%\end{array}$ & $\begin{array}{l}+0.6 \% \\
-1.0 \%\end{array}$ & & $\begin{array}{l}+1.9 \% \\
-1.8 \%\end{array}$ \\
\hline$K^{\mathrm{b}}=$ & 1.03878 & 1.02175 & 1.05479 & 1.03468 & & 0.99651 \\
\hline
\end{tabular}

${ }^{\mathrm{a}}$ The best precision is obtained when the entire range on the centimeter delay dial is used: good use, $6-10$; fair use, 3-6; poor use, 0-3.

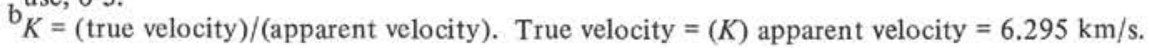

TABLE 9

Uncorrected Velocities $(\mathrm{km} / \mathrm{s})$ through Brass Sonic Semi-Standards

\begin{tabular}{|c|c|c|c|c|c|c|}
\hline \multirow{3}{*}{$\begin{array}{l}\text { Scope Settinga } \\
\text { Semi-Standard } \\
\text { Thickness }\end{array}$} & \multicolumn{2}{|l|}{$1.0 \mu \mathrm{s} / \mathrm{cm}$} & \multicolumn{2}{|c|}{$2.0 \mu \mathrm{s} / \mathrm{cm}$} & \multicolumn{2}{|r|}{$5.0 \mu \mathrm{s} / \mathrm{cm}$} \\
\hline & & & & & & \\
\hline & $2.54 \mathrm{~cm}$ & $5.00 \mathrm{~cm}$ & $2.54 \mathrm{~cm}$ & $5.00 \mathrm{~cm}$ & $2.54 \mathrm{~cm}$ & $5.00 \mathrm{~cm}$ \\
\hline & $\begin{array}{c}\text { (Fair use of } \\
\text { centimeter delay) }\end{array}$ & & $\begin{array}{c}\text { (Poor use of } \\
\text { centimeter delay) }\end{array}$ & $\begin{array}{c}\text { (Fair use of } \\
\text { centimeter delay) }\end{array}$ & & $\begin{array}{c}\text { (Poor use of } \\
\text { centimeter delay) }\end{array}$ \\
\hline & 4.361 & & 4.384 & 4.346 & & 4.424 \\
\hline & 4.468 & & 4.372 & 4.297 & & 4.463 \\
\hline & 4.424 & & 4.377 & 4.264 & & 4.509 \\
\hline & 4.430 & & 4.400 & 4.310 & & 4.430 \\
\hline & 4.465 & & 4.369 & 4.326 & & 4.454 \\
\hline & 4.395 & & 4.375 & 4.303 & & 4.438 \\
\hline & 4.396 & & & 4.287 & & 4.496 \\
\hline & 4.444 & & 4.407 & 4.300 & & 4.420 \\
\hline & 4.373 & & 4.384 & 4.273 & & 4.428 \\
\hline & 4.343 & & 4.384 & 4.289 & & 4.414 \\
\hline Average & 4.410 & & 4.384 & 4.300 & & 4.448 \\
\hline Range & $\begin{array}{l}+1.3 \% \\
-1.5 \%\end{array}$ & & $\begin{array}{l}+0.5 \% \\
-0.3 \%\end{array}$ & $\begin{array}{l}+1.1 \% \\
-0.8 \%\end{array}$ & & $\begin{array}{l}+1.4 \% \\
-0.8 \%\end{array}$ \\
\hline$K^{\mathrm{b}}=$ & 1.02698 & & 1.03307 & 1.05326 & & 1.01821 \\
\hline
\end{tabular}

a The best precision is obtained when the entire range on the centimeter delay dial is used: good use, 6-10, fair use,

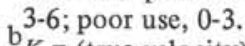

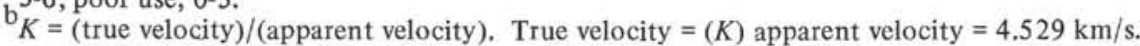

each $\mu \mathrm{s} / \mathrm{cm}$ setting on the oscilloscope, as follows (Table 12):

(average apparent velocity $\times(K)=$ true velocity of semi-standards

\section{VANE SHEAR MEASUREMENTS}

We attempted to take vane shear measurements on relatively undisturbed clay samples. Vane shear strength is defined as the maximum torque applied to a vane in a clayey sample before failure of the clay. Failure occurs around the cylindrical surface area of the vane, and the final shear strength is the force $(\mathrm{kg})$ per unit area $\left(\mathrm{cm}^{2}\right)$ of the cylindrical vane. The sample is considered to be undrained. Theoretically, shear strength comprises cohesional and frictional components. The vane shear device theoretically (not completely in reality) measures the cohesional component of the total shear strength of the sediment.

On Leg 50 we measured vane shear strength with the DSDP Wykeham Farrance Laboratory Vane apparatus. The equipment, techniques, and calibrations are dis- 
TABLE 10

Uncorrected Velocities $(\mathrm{km} / \mathrm{s})$ through Lucite Sonic Semi-Standards

\begin{tabular}{|c|c|c|c|c|c|c|}
\hline \multirow{2}{*}{$\begin{array}{c}\text { Scope Settinga } \\
\text { Semi-Standard } \\
\text { Thickness } \\
\end{array}$} & \multicolumn{2}{|c|}{$1.0 \mu \mathrm{s} / \mathrm{cm}$} & \multicolumn{2}{|c|}{$2.0 \mu \mathrm{s} / \mathrm{cm}$} & \multicolumn{2}{|r|}{$5.0 \mu \mathrm{s} / \mathrm{cm}$} \\
\hline & $2.54 \mathrm{~cm}$ & $5.00 \mathrm{~cm}$ & $2.54 \mathrm{~cm}$ & $5.00 \mathrm{~cm}$ & $2.54 \mathrm{~cm}$ & $5.00 \mathrm{~cm}$ \\
\hline & $\begin{array}{c}\text { (Good use of } \\
\text { centimeter delay) }\end{array}$ & & $\begin{array}{c}\text { (Fair use of } \\
\text { centimeter delay) }\end{array}$ & $\begin{array}{c}\text { (Good use of } \\
\text { centimeter delay) }\end{array}$ & & $\begin{array}{c}\text { (Fair use of } \\
\text { centimeter delay) }\end{array}$ \\
\hline & $\begin{array}{l}2.689 \\
2.714 \\
2.714 \\
2.713 \\
2.714 \\
2.718 \\
2.708 \\
2.702 \\
2.717 \\
2.726\end{array}$ & & $\begin{array}{l}2.650 \\
2.656 \\
2.665 \\
2.645 \\
2.662 \\
2.668 \\
2.660 \\
2.646 \\
2.635 \\
2.675\end{array}$ & $\begin{array}{l}2.664 \\
2.671 \\
2.670 \\
2.666 \\
2.673 \\
2.665 \\
2.653 \\
2.668 \\
2.665 \\
2.647\end{array}$ & & $\begin{array}{l}2.713 \\
2.727 \\
2.739 \\
2.722 \\
2.739 \\
2.773 \\
2.745 \\
2.730 \\
2.744 \\
2.736\end{array}$ \\
\hline Average & 2.712 & & 2.656 & 2.664 & & 2.737 \\
\hline Range & $\begin{array}{l}+0.5 \% \\
-0.8 \%\end{array}$ & & $\begin{array}{l}+0.7 \% \\
-0.4 \%\end{array}$ & $\begin{array}{l}+0.3 \% \\
-0.7 \%\end{array}$ & & $\begin{array}{l}+1.3 \% \\
-0.9 \%\end{array}$ \\
\hline$K^{\mathrm{b}}=$ & 1.01180 & & 1.03313 & 1.03003 & & 1.00256 \\
\hline
\end{tabular}

TABLE 11

Uncorrected Velocities $(\mathrm{km} / \mathrm{s})$ through Distilled Water $\left(22^{\circ} \mathrm{C}, 1.491 \mathrm{~km} / \mathrm{s}\right)$ Sonic Standard

\begin{tabular}{lccc}
\hline $\begin{array}{c}\text { Scope Setting } \\
\text { Semi-Standard } \\
\text { Thickness }\end{array}$ & $1.0 \mu \mathrm{s} / \mathrm{cm}$ & $2.0 \mu \mathrm{s} / \mathrm{cm}$ & $5.0 \mu \mathrm{s} / \mathrm{cm}$ \\
\hline & $0.4 \mathrm{~cm}$ & $1.9 \mathrm{~cm}$ & $1.8 \mathrm{~cm}$ \\
\hline & $\begin{array}{c}\text { (Poor use of } \\
\text { centimeter delay) }\end{array}$ & $\begin{array}{c}\text { (Good use of } \\
\text { centimeter delay) }\end{array}$ & $\begin{array}{c}\text { (Poor use of } \\
\text { centimeter delay) }\end{array}$ \\
& 1.477 & 1.461 & 1.478 \\
& 1.454 & 1.459 & 1.481 \\
1.462 & 1.457 & 1.472 \\
& 1.478 & 1.443 & 1.475 \\
& 1.505 & 1.460 & 1.473 \\
& 1.492 & 1.455 & 1.489 \\
& 1.464 & 1.454 & 1.477 \\
Average & 1.457 & 1.456 & 1.491 \\
Range & 1.501 & 1.455 & 1.488 \\
& 1.477 & 1.456 & 1.488 \\
$K \mathrm{~b}=$ & $+1.9 \%$ & $+0.4 \%$ & 1.481 \\
& $-1.6 \%$ & $-0.5 \%$ & $+0.7 \%$ \\
& 1.00948 & 1.02404 & $-0.7 \%$ \\
\hline
\end{tabular}

${ }^{\mathrm{a}}$ The best precision is obtained when the entire range on the centimeter belay dial is used: good use, 6-10; fair use, 3-6; poor use, 0-3.

$K=$ (true velocity) $/$ (apparent velocity). True velocity $=(K)$ apparent velocity $=1.491 \mathrm{~km} / \mathrm{s}$.

cussed in Boyce (1977); only changes from Boyce (1977) and other pertinent information are discussed here. We used a $1.278 \times 1.278 \mathrm{~cm}$ vane (number 1) and buried it about $0.5 \mathrm{~cm}$ on top and bottom of the sample. Because we split the cores to find a suitable type of sediment, we inserted the vane parallel to bedding. We applied torque to the spring (number 4 ) at a rate of $89^{\circ}$ per minute. The remolded test was done immediately after we rotated the vane, while in the sample, 10 times.

\section{THERMAL CONDUCTIVITY}

According to Langseth (1965), "The heat flux at the surface can be determined by measuring the tempera-
TABLE 12

Calculation of Final Correction Factors (K) for Each $\mu \mathrm{s} / \mathrm{cm}$ Setting on DSDP Tektronix 561A Oscilloscope

\begin{tabular}{|c|c|c|c|c|c|c|}
\hline \multirow{2}{*}{$\begin{array}{l}\text { Scope Settinga } \\
\text { Semi-Standard } \\
\text { Thickness }\end{array}$} & \multicolumn{2}{|c|}{$1.0 \mu \mathrm{s} / \mathrm{cm}$} & \multicolumn{2}{|c|}{$2.0 \mu \mathrm{s} / \mathrm{cm}$} & \multicolumn{2}{|c|}{$5.0 \mu \mathrm{s} / \mathrm{cm}$} \\
\hline & $<2.54 \mathrm{~cm}$ & $5.00 \mathrm{~cm}$ & $\leqslant 2.54 \mathrm{~cm}$ & $5.00 \mathrm{~cm}$ & $<2.54 \mathrm{~cm}$ & $5.00 \mathrm{~cm}$ \\
\hline $\begin{array}{l}\text { Water } \\
(0.4 \mathrm{~cm})\end{array}$ & $\begin{array}{l}1.00948^{b} \\
\text { (deleted) }\end{array}$ & & & & & \\
\hline $\begin{array}{l}\text { Water } \\
(1.9 \mathrm{~cm})\end{array}$ & & & $1.02404^{\mathrm{c}}$ & & $1.00675^{\mathrm{b}}$ & \\
\hline Lucite & $1.01180^{c}$ & & 1.03313 & $1.03003 \mathrm{c}$ & & $1.00256 \mathrm{~d}$ \\
\hline Brass & $1.02698^{\mathrm{d}}$ & & $1.03307^{b}$ & $1.05326 \mathrm{~d}$ & & $1.01821^{b}$ \\
\hline Aluminum & $1.03878^{d}$ & $1.02175^{\mathrm{c}}$ & $1.05479 \mathrm{~b}$ & $1.03468 \mathrm{~d}$ & $0.99651^{b}$ & \\
\hline Ea. Avg. & 1.02585 & 1.02175 & 1.03626 & 1.03932 & 1.00163 & 1.01039 \\
\hline Mean $K^{\mathrm{e}}$ & \multicolumn{2}{|c|}{1.02380} & \multicolumn{2}{|c|}{1.03779} & \multicolumn{2}{|c|}{1.00601} \\
\hline
\end{tabular}

ture gradient and multiplying by the 'thermal' conductivity of the material between the two points of measurement." The extent to which the temperature distribution at depth can be extrapolated via thermal-conductivity measurements depends on (1) whether or not the interval of temperature measurement is free from the flow of mass (circulating interstitial water, for example, would greatly disturb the conductive heat transfer); (2) whether or not the conductivities of the rocks at the site are homogeneous enough to allow meaningful extrapolations of the observed heat flux; and (3) whether or not the actual conductivity measurements are representative of in situ conditions.

Heat-conductivity measurements conducted in conjunction with continuous porosity measurements such as GRAPE and well-log density data allow us to determine an approximate average heat conductivity that we 
must know in order to extrapolate temperatures with increasing depth.

The transient-needle method was used aboard the Glomar Challenger to measure the heat conductivity of the sediments. This equipment was set up by Dr. Albert Ericson. This needle is $6.4 \mathrm{~cm}$ long and $1.0 \mathrm{~mm}$ in diameter; it contains a heater and thermistor. The rate at which the sediment dissapates the heat is a function of its thermal conductivity. This method is described in detail by von Herzen and Maxwell (1959) and Langseth (1965). The method has a reproducibility of about 2.5 per cent.

The heat conductivities are reported in the site chapters (this volume) at ambient laboratory temperatures and pressures.

\section{REFERENCES}

Boyce, R. E., 1973. Appendix I. Physical property methods. In Edgar, N. T., Saunders, J. B., et al., Initial Reports of the Deep Sea Drilling Project, v. 15, Pt. 2: Washington (U.S. Government Printing Office), p. 1115-1127.
1976. Definitions and laboratory techniques of the compressional sound velocity parameters and wet-water content, wet-bulk density, and porosity parameters by gravimetric and gamma ray attenuation techniques. In Schlanger, S. O., Jackson, E. D., et al., Initial Reports of the Deep Sea Drilling Project, v. 33: Washington (U.S. Government Printing Office), p. 931-988.

1977. Deep Sea Drilling Project procedures for shear strength measurement of clayey sediment using Modified Wykeham Farrance Laboratory Vane Apparatus. In Talwani, M., Udintsev, G., et al., Initial Reports of the Deep Sea Drilling Project, v. 38: Washington (U.S. Government Printing Office), p. 1059-1068.

Evans, H. B., 1965. GRAPE-A device for continuous determination of material density and porosity; SPWIA Logging Symp., 6th Ann., Trans., Dallas, Texas, v. 2, p. B1-B25.

Langseth, M. G., 1965. Techniques of measuring heat flow through the ocean floor. In Terrestrial Heat Flow: Am. Geophys. Union Monograph 8, p. 58.

Mottaboni, P. and Schreiber, E., 1967. Method of pulse transmission measurements for determining sound velocities, $J$. Geophys. Res., v. 72, p. 5160 .

von Herzen, R. P. and Maxwell, A. E., 1959. The measurement of thermal conductivity of deep-sea sediments by a needle-probe method, J. Geophys. Res., v. 64, p. 1557. 